\title{
Estimativa do risco de infecção tuberculosa em populações vacinadas pelo $B C G^{*}$
}

\section{Determining the risk of tuberculosis infection in BCG-vaccinated populations}

\author{
Gilberto Ribeiro Arantes**, Stella Maria C. Nardy"*, Rosa M.G. Weiler"**, Marília Belluomini", \\ Péricles A. Nogueira**
}

\begin{abstract}
ARANTES, G. R. et al. Estimativa do risco de infecção tuberculosa em populações vacinadas pelo BCG. Rev. Saúde públ., S. Paulo, 26: 96-107, 1992. A revacinação de escolares com BCG, capaz de restaurar a alergia remanescente de vacinação realizada nos primeiros meses de vida, porém incapaz de modificar a alergia devida à infecção pelo $M$. luberculosis, possibilitaria a quantificação da parcela dessa população infectada pelo bacilo de Koch. Foi desenvolvida pesquisa com o objetivo de avaliar a aplicabilidade desses pressupostos na estimativa do risco de infecção tuberculosa em áreas sob elevada cobertura com BCG. A população de estudo foi constituída por escolares com 6 a 9 anos de idade freqüentando escolas municipais da zona leste da cidade de São Paulo, durante o primeiro semestre letivo de 1988. De 11.455 vacinados, apenas 7.470 foram submetidos ao teste tuberculínico, revacinados em seguida e retestados dez semanas depois. Destes, 3.314 tinham sido vacinados no primeiro trimestre de vida com meia dose c os demais 4.156 receberam dose plena acima dessa idade ( $75 \%$ no primeiro ano de vida, $20 \%$ no segundo e $5 \%$ no terceiro). A contagem dos infectados, pelo confronto dos resultados pré e pós vacinais $\mathrm{cm}$ tabelas de correlação, foi realizada segundo os critérios do método original e modificação introduzida pelos autores, separadamente para os vacinados no primeiro trimestre de vida e após essa idade. O risco de infecção foi, respectivamente, $0,35 \%$ e $0,37 \%$ com o critério original e $0,45 \%$ e $0,49 \%$ com o modilicado. O referencial médio disponível para a área estudada, estimado por outros mélodos, foi $0,55 \%$. As diferenças entre critérios e idades e destes com o referencial não foram significantes $(P>0,05)$. Os resultados sugerem que o método é aplicável para a cstimativa do risco de infeç̧ão tuberculosa na idade escolar, em vacinados com BCG no primeiro ano de vida, com dose plena de vacina.
\end{abstract}

Descritores: Tuberculose, prevenção e controle. Vacina BCG. Vigilância epidemilógica.

\section{Introduçăo}

As reações cruzadas devidas a infecções por micobactérias alípicas diminuem a eficácia do teste tuberculínico padronizado ${ }^{18}$, comprometendo seu uso na vigilância da tuberculose. Esse efeito ć tanto mais acentuado quanto mais clevada for a proporção dessas infecçð̃es $\mathrm{cm}$ relação à prevalência da infecção específica ${ }^{1}$.

O mesmo acontece em áreas extensamente vacinadas com BCG intra-dérmico cuja influência

* Pesquisa financiada pelo Instituto de Saúde da Secretaria da Saúde do Estado de São Paulo com recursos do Programa Metropolitano de Saúde/Banco Mundial, Processo SS $1881 / 86$

* Departamento de Epiderniologia da Faculdade de Saúde Pública da Universidade de São Paulo - São Paulo, SP Brasil.

*** Secretaria de Estado da Saúde de São Paulo - São Paulo, SP-Brasil.

Separatas/Reprints: G. R. Arantes - Av. Dr. Amaldo, 715 01246 - 904 - São Paulo, Sl’ - Brasil.

Publicação financiada pcla FAPESP. Processo Saúde Colctiva 91/4994-0 sobre o perfil tuberculínico pode perdurar até a idade escolar, mesmo quando a vacina é aplicada nos primeiros meses de vida ${ }^{3}$.

Em siluações como essas, embora não seja possível distinguir indivíduos infectados dos não infectados, a prevalência daqueles pode ser estimada decompondo-se matematicamente o perfil tuberculínico dessas populações ${ }^{4,26}$.

$\mathrm{Na}$ falta de uma tuberculina específica para o BCG, Dam e Hitze $e^{5}$ propuseram um método que, ao lado da cstimativa da proporção de infectados, possibilitaria a identificação dos individuos atingidos. Tal mélodo ć bascado em conhecimentos disponíveis sobre as inter-relações entre as hipersensibilidades devidas aos diversos agentes micobacterianos envolvidos na questão. Assim, (1) a alcrgia induzida por infecções atípicas aparentemente enfraquece com o passar do tempo, tal como acontece com a alcrgia induzida pelo $\mathrm{BCG}^{9,10}$, podendo ser restaurada pelo teste tuberculínico $^{6,15,20}$; (2) em contrapartida, a alergia resultante de infecção pelo $M$. tuberculosis é muito estávcl ${ }^{16}$, por isso nāo sujeita ao cfcito restaurador do teste; (3) a vacina BCG não incrementa a alergia tuberculinica de pessoas anteriormente infecta- 
das pelo bacilo de Koch ${ }^{16}$; (4) a vacina BCG em infectados por micobactérias atípicas gera um perfil idêntico ao que resulta da vacinação de não infectados por esses microorganismos ${ }^{23}$.

Por conseqüência, a relação entre duas reações tuberculínicas sucessivas em um mesmo indivíduo possibilitaria a distinção entre reaçães específicas e inespecíficas, sob determinadas condiçōes: numa população constituída por uma mistura de não infectados, infectados por outras micobactérias e infectados pelo bacilo de Koch, a aplicação de uma dose de BCG potente reforçaria a alergia induzida pela micobactéria atípica, ou os resquícios de uma alergia vacinal anterior, sem entretanto modificar as reaçōes específicas. Em outras palavras, as reaçöes não incrementadas identificariam os indivíduos infectados pelo bacilo da tuberculose.

Os autores da proposta ${ }^{5}$ ressalvam porém que, apesar das semelhanças entre as alergias induzidas pelo BCG e pelas micobactérias atípicas, elas são quantitativamente diferentes, atribuindo-se maior intensidade ao BCG.

Por esse motivo, uma alergia vacinal ainda intensa poderá não ser reforçada por uma nova dose de vacina ${ }^{19}$, levando com isso a uma falsa interpretação positiva. Uma possivel exceção seria a situação na qual a vacinação é realizada com dose reduzida, em recém-nascidos, os quais costumam apresentar respostas menos intensas ${ }^{8}$ mesmo quando vacinados com dose plena ${ }^{25}$; $\mathrm{cm}$ revacinados à idade escolar espera-se que ocorra um incremento na alergia capaz de superar o erro experimental do teste.

No Brasil vigorou até 1983 norma técnica estabelecendo a aplicação de $1 / 2$ dose em crianças com menos de 90 dias de vida11. Com o que, ainda seria possível encontrar em 1988 crianças à idade escolar que atendessem ao pré-requisito estabelecido por Dam e Hitze 5 .

Isto posto, a presente pesquisa foi planejada com o objetivo geral de analisar a aplicabilidade do método com vistas à estimativa do risco de infecção tuberculosa e com os objclivos específicos de: 1 - comparar os resultados de sua aplicação em escolares primo-vacinados no primeiro trimestre de vida com escolares primovacinados depois dessa idade; 2 - confrontar os riscos de infecção tuberculosa assim estimados com outras estimativas disponíveis para a área epidemiológica estudada.

\section{Material e Método}

A população de estudo foi constituída por alunos da primeira série de 53 das 54 escolas municipais de primeiro grau localizadas na zona leste da cidade de São Paulo, onde é elevada a proporção de familias de baixa renda24. No início do primeiro semestre escolar de 1988, após trabalho preparatório realizado por educadoras de saúde pública, foram anotados em fichas individuais numeradas os dados de identificação das crianças, data de nascimento e data da vacinação (retirada da cadcrneta de vacinação ou documento equivalente). Uma equipe constituída por $5 \mathrm{en}$ fermeiros e 2 auxiliares técnicos, todos com grande experiência nesse tipo de pesquisa, supervisionados por enfermeira de saúde pública*, encarregou-se da pesquisa da cicatriz vacinal em ambos os braços e da aplicação e leitura dos testes tuberculínicos, segundo a norma técnica em vigor ${ }^{12}$. No dia da leitura foi aplicada uma dose de $B C G$, independentemente do antecedente vacinal. Decorrido um período que variou de 10 a 12 semanas, foi aplicado novo teste de Mantoux, usando-se duplicatas das fichas para anotaçס̄es; a fim de que os leitores desconhecessem o resultado da primeira leitura.

Das fichas, as informações foram transferidas para arquivo DBase III Plus e processadas em microcomputador de 16 bits com memória RAM de 20 megabytcs.

Dos parlicipantes com $6 \vdash 9$ anos de idade que se submeteram as duas leituras, separaram-se os vacinados no primeiro trimestre de vida e os vacinados acima dessa idade, doravante denominados primeira e segunda idade.

A identificaçăo e contagem dos infectados foi feita separadamente para cada uma dessas idades, confrontando-se as reações pré e pós revacinação em tabelas de correlação de acordo com o método proposto :

a) eliminadas do numerador e denominador as reaçōes zero ou muito pequenas (até $3 \mathrm{~mm}$ ) em ambos os testes, observadas sobre a diagonal ou abaixo dela, e as reações mensuráveis ao primeiro teste com resultado zcro ao segundo;

b) contados os individuos cujas reações foram iguais nos dois testes (sobre a diagonal); aqueles cuja segunda reação foi menor do que a primeira (abaixo da diagonal); e os indivíduos com reações maiores ao segundo teste (acima da diagonal), porém limitados pelo número contado abaixo da diagonal na respectiva linha.

Pclos motivos que scrão expostos na discussão, foi realizada uma contagem altcmativa dos indivíduos com reações maiores ao segundo teste, limitados porém pelo afastamento observado abaixo da diagonal, na respectiva linha.

* Enf. Janctte Nassar, do Grupo de Programa de Tuberculose da SES de S. Paulo. 
O risco anual de infecção foi calculado pela expressão $\mathrm{r}=1 \cdot\left(\mathrm{N}_{\mathfrak{n}}\right) 1 / \mathrm{n}$, utilizada cm pesquisa anterior ${ }^{2}$.

\section{Resultados}

Dos 12.442 participantes iniciais com $6 \vdash 9$ anos de idade, $11.455(92 \%)$ estavam presentes no dia da primeira leitura do teste tuberculínico, sendo que apenas 30 deixaram de receber nova dose de vacina. Estavam presentes na data do reteste $9.314(75 \%)$, dos quais $8.329(67 \%)$ compareceram no dia da leitura.
As perdas entre a aplicação e leitura foram semelhantes nas duas ocasioes ( 8 a 10\%). Dos 8.329 que passaram pelas duas etapas, $7.470(90 \%)$ tinham cicauriz vacinal. Destes, 3.314 foram vacinados na primeira idade e 4.156 na segunda; dos últimos, $20 \%$ no segundo ano de vida e $5 \%$ no terceiro.

Na Tabcla 1 é apresentada a correlação entre os resultados pré e pós vacinais nos 3.314 escolares vacinados na primeira idade.

Foram desprezados, para fins de cálculos, os valores sublinhados, em número de 44 , subtraídos dos 3.314 escolares. A contagem dos infectados pelo critério original revelou 32 indivíduos abaixo da diagonal, 18 sobre ela e 26 acima, perfazendo

Tabela 1. Correlação entre os diâmetros pré e pós vacinais em escolares com 6 a 8 anos de idade, primovacinados com BCG no primeiro trimestre de vida, zona leste da Cidade de S. Paulo, 1988.

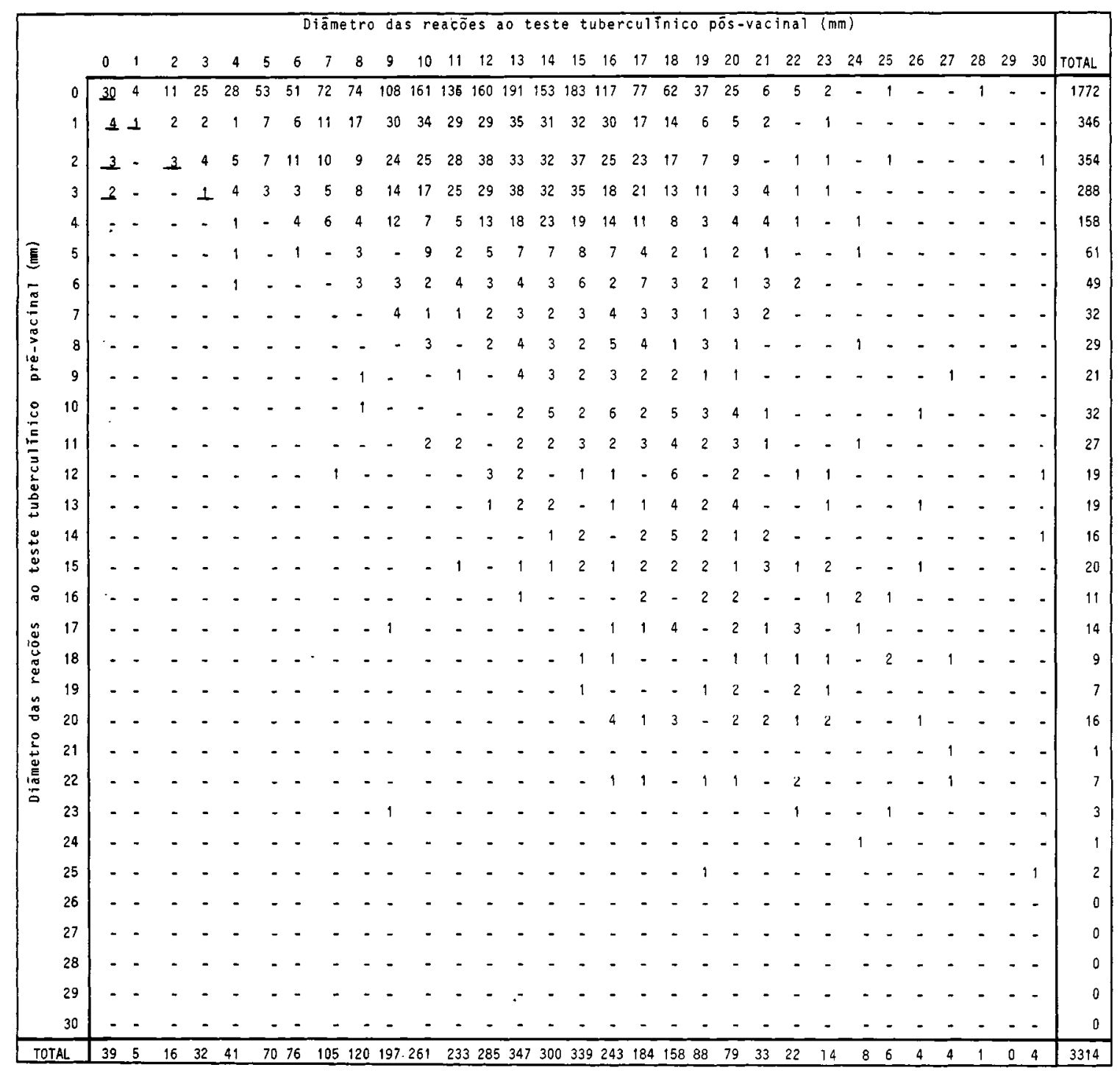


76 em 3.270 (2,32\%); pelo critério alternativo, os infectados acima da diagonal foram 47 , fazendo com que o total aumentasse para $97 \mathrm{~cm} 3.270$ $(2,97 \%)$.

A distribuição marginal à direita da Tabcla 1 (somatória das linhas) representa as reações ao primeiro Mantoux, cujo perfil é mostrado na FIGURA 1A. A distribuição marginal inferior representa as reações ao segundo Mantoux e o scu perfil é apresentado em B, NA FIGURA 1.

Na Tabela 2, que se refere aos 4.156 vacinados na segunda idade, os valores descontados (sublinhados) totalizaram 46 , diminuídos dos 4.156. A contagem dos infectados revelou 58,18 e 49 in- divíduos, respectivamente abaixo, sobre e acima da diagonal, tolalizando $125 \mathrm{em} 4.110$ (3,04\%); pclo critério altcrnativo, os infectados passaram a ser $146 \mathrm{~cm} 4.110(4,04 \%)$.

As Tabclas $3 \mathrm{e} 4$ apresentam as distribuiçōes das diferenças entre a primeira e a segunda leituras do Mantoux relativas aos infectados identificados na sub-população vacinada na primeira idade, segundo os critérios original e alternativo; os respectivos perfis tuberculínicos aparecem na FIGURA 3 A e B. As Tabclas 5 e 6 reproduzem essas distribuições na subpopulação vacinada na segunda idade.

$\mathrm{Na}$ Tabela 7 constam as estimativas do risco anual de infecção tuberculosa nas duas sub-

Tabela 2. Correlação entre os diâmetros pré e pós vacinais em escolares com 6 a 8 anos de idade, primovacinados com BCG após o primeiro trimestre de vida, zona leste da Cidade de S. Paulo, 1988.

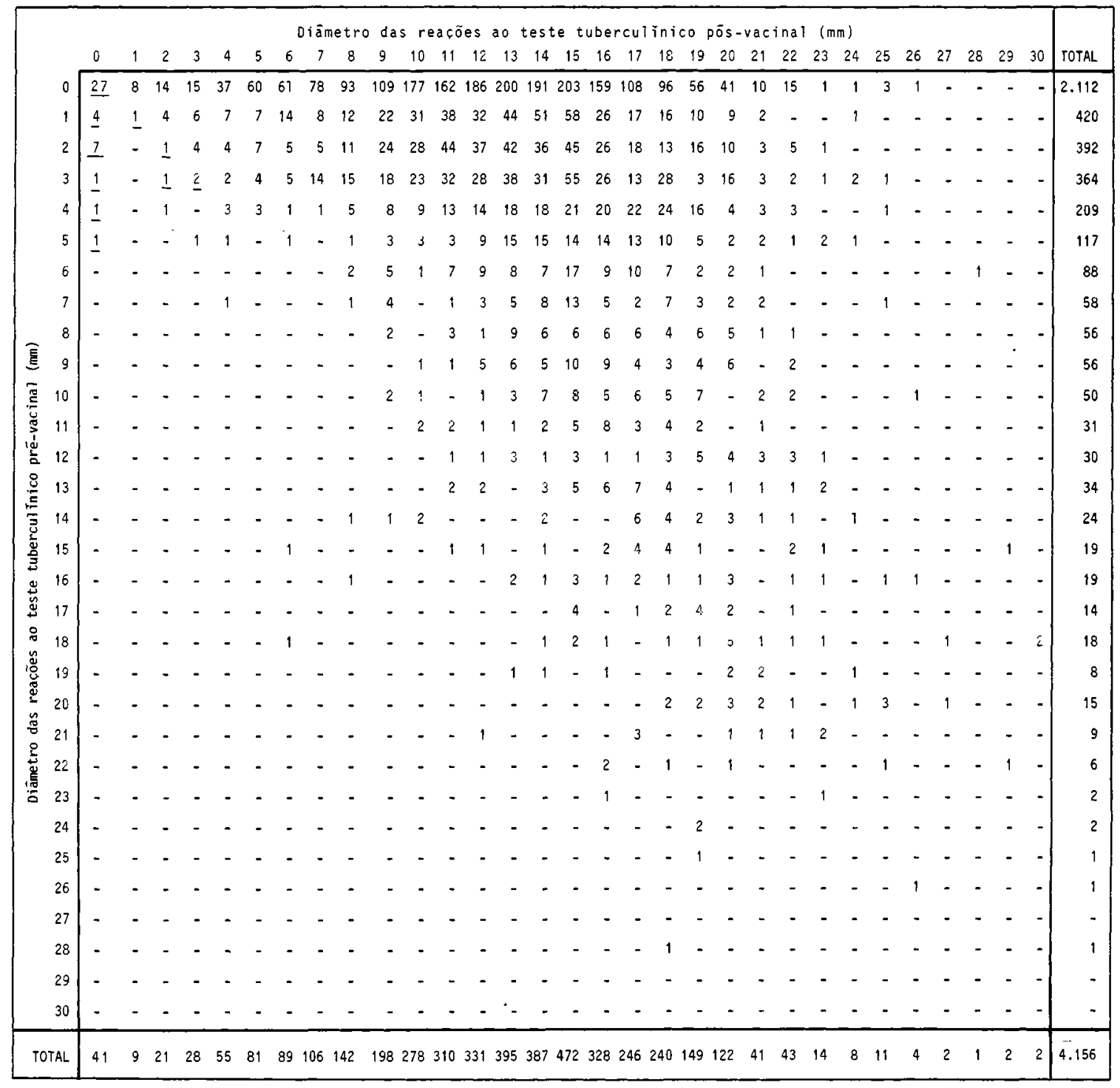


Tabela 3. Distribuição das diferenças de leitura do primeiro para o segundo teste tuberculínico, infectados definidos pelo critério original. Vacinados no primeiro trimestre de vida.

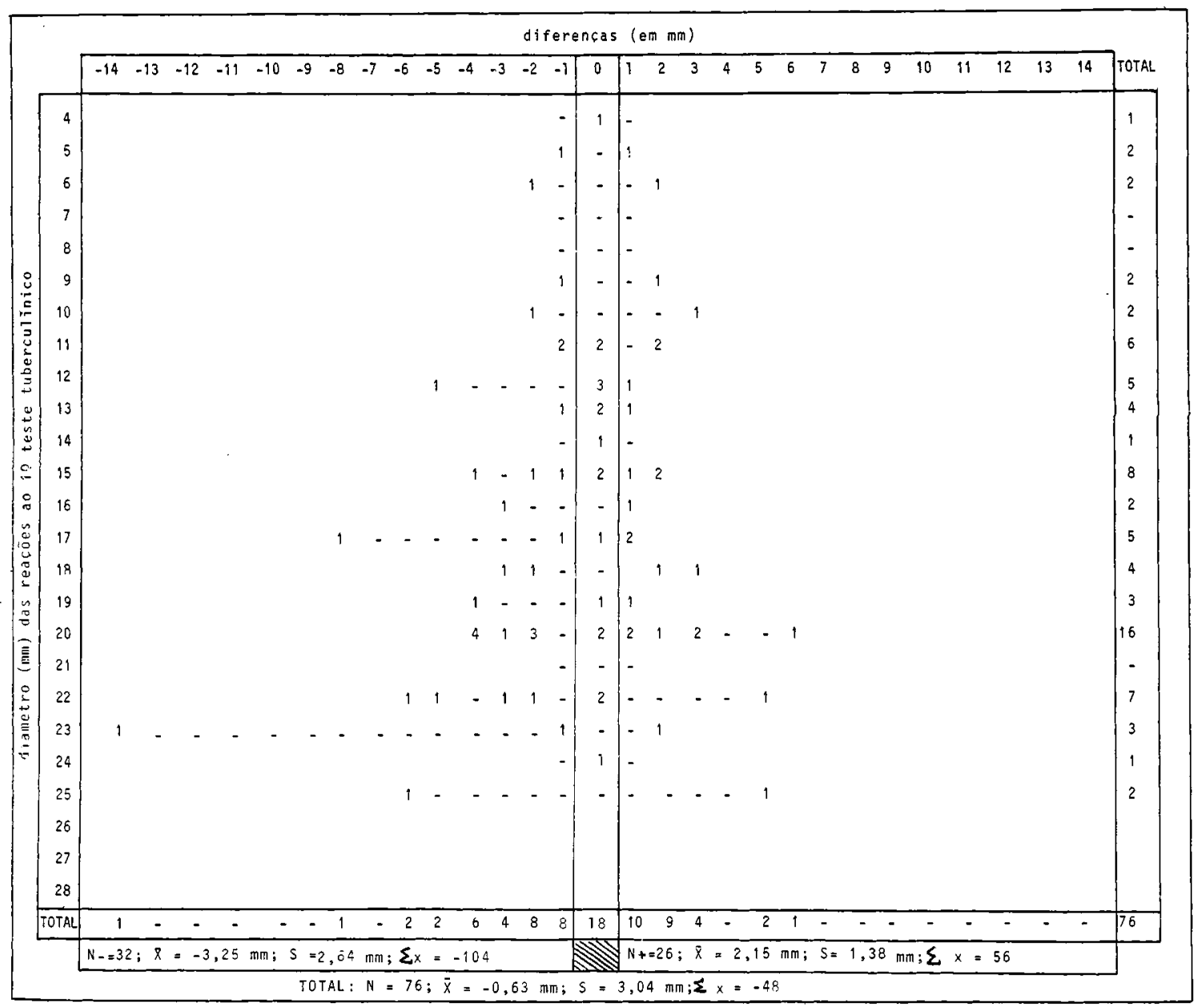


Tabela 4. Distribuiçăo das diferenças de leitura do primeiro para o segundo teste tuberculínico, infectados definidos pelo critério modificado. Vacinados no primeiro trimestre de vida.

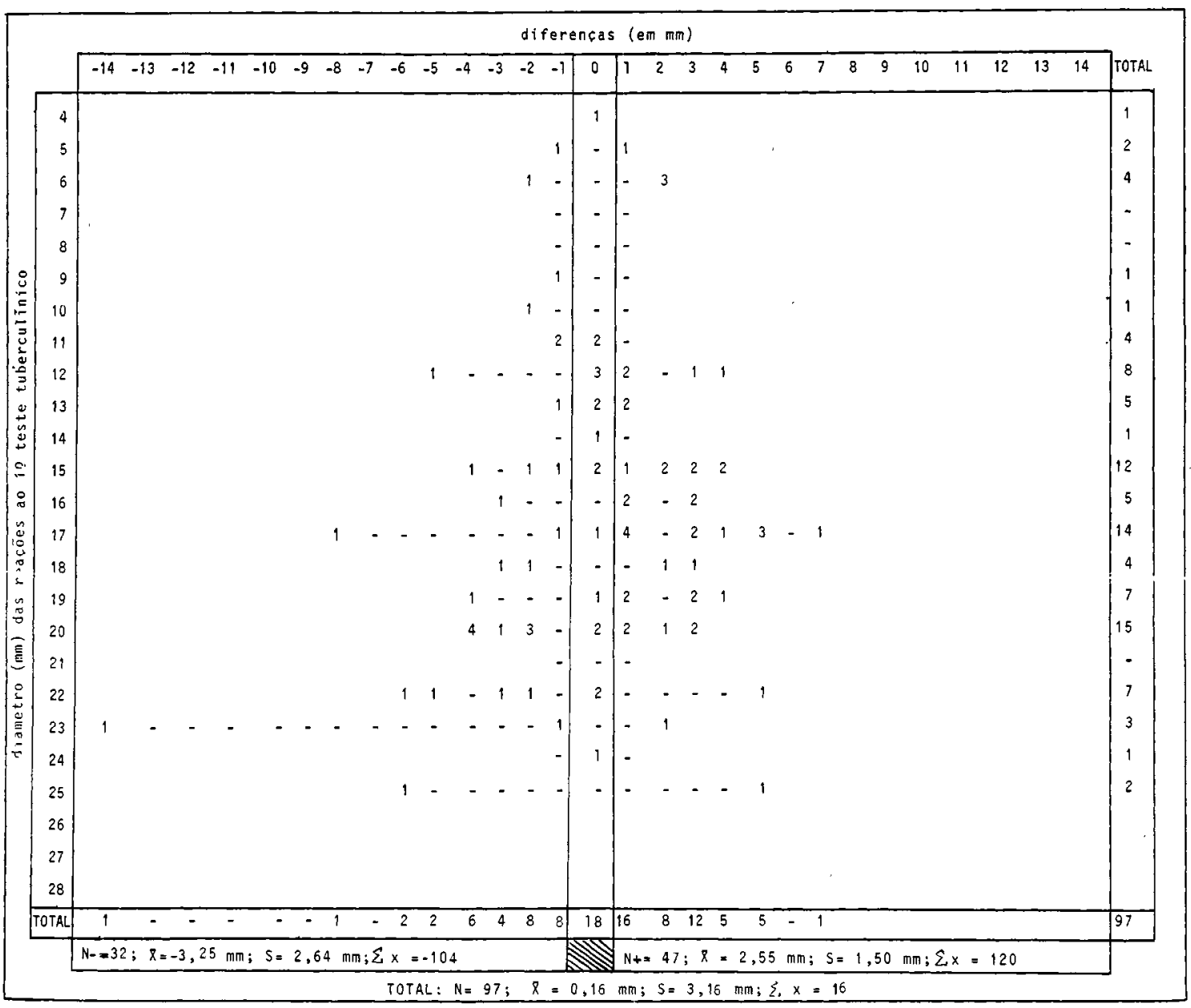


Tabela 5. Distribuição das diferenças de leitura do primeiro para o segundo teste tuberculínico, infectados definidos pelo critério original. Vacinados depois do primeiro trimestre.

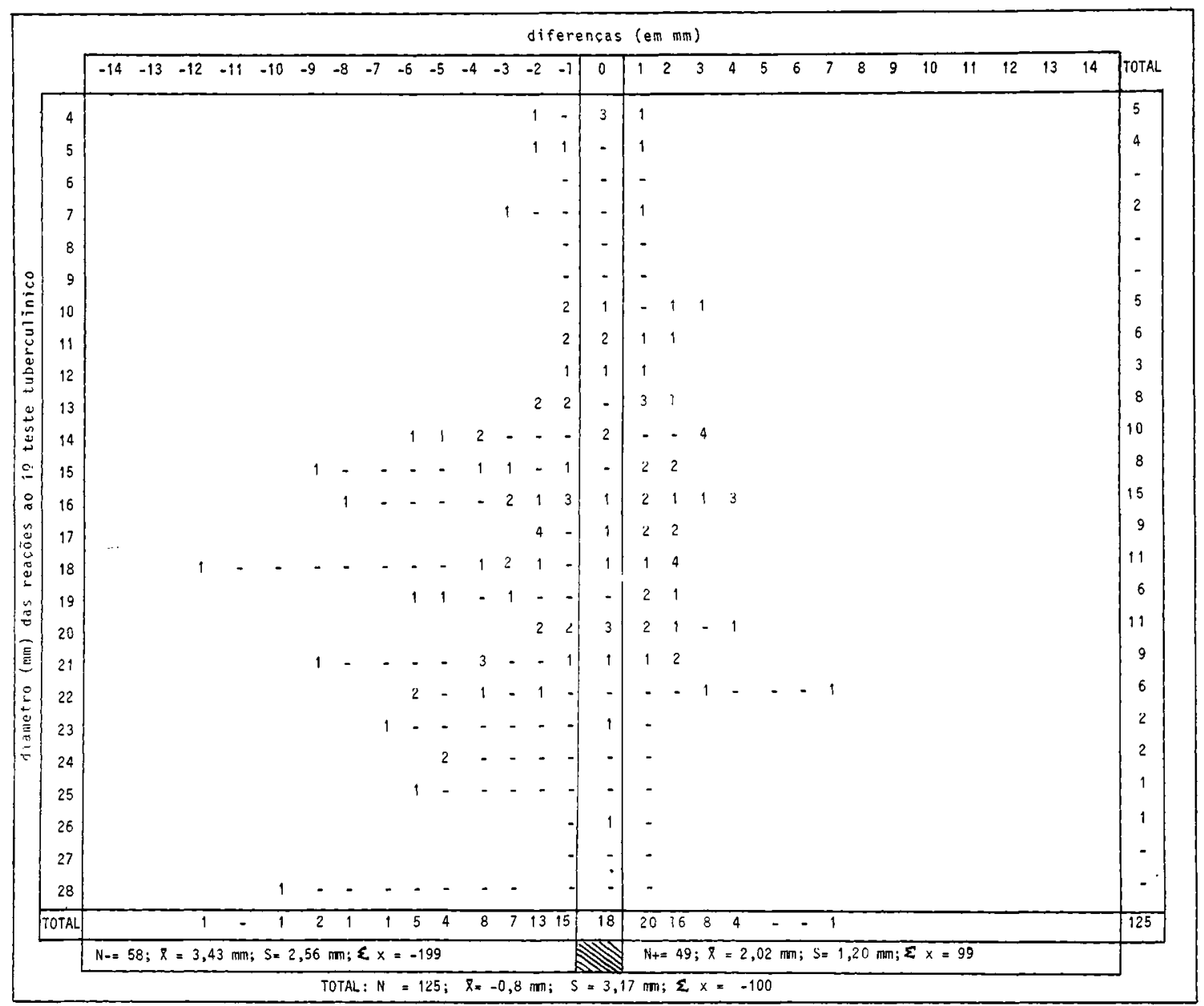


Tabela 6. Distribuição das diferenças de leitura do primeiro para o segundo teste tuberculínico, infectados definidos pelo critério modificado. Vacinados depois do primeiro trimestre.

\begin{tabular}{|c|c|c|c|c|c|c|c|c|c|c|c|c|c|c|c|c|c|c|c|c|c|c|c|c|c|c|c|c|c|c|c|c|c|}
\hline & \multicolumn{32}{|c|}{ diferencas (em $\mathrm{mm}$ ) } \\
\hline & & -14 & -13 & -12 & -11 & -1 & 10 & -9 & -8 & -7 & -8 & 6 & -5 & -4 & -3 & -2 & -1 & 0 & 1 & 2 & 3 & 4 & 5 & 6 & 6. & 7 & 8 & 9 & 10 & 11 & 12 & $\begin{array}{ll}13 & 14\end{array}$ & TOTAL \\
\hline & 4 & & & & & & & & & & & & & & & 1 & -1 & 3 & 3 & 1 & & & & & & & & & & & & & 8 \\
\hline & 5 & & & & & & & & & & & & & & & & 1 & - & 1 & & & & & & & & & & & & & & 3 \\
\hline & 6 & & & & & & & & & & & & & & & & - & - & - & & & & & & & & & & & & & & - \\
\hline & 7 & & & & & & & & & & & & & & 1 & - & - & - & 1 & 4 & & & & & & & & & & & & & 6 \\
\hline & 8 & & & & & & & & & & & & & & & & - & - & - & & & & & & & & & & & & & & - \\
\hline 8 & 9 & & & & & & & & & & & & & & & & - & - & - & & & & & & & & & & & & & & - \\
\hline$E$ & 10 & & & & & & & & & & & & & & & & 2 & 1 & - & & & & & & & & & & & & & & 3 \\
\hline $\bar{z}$ & 11 & & & & & & & & & & & & & & & & 2 & 2 & 1 & & & & & & & & & & & & & & 5 \\
\hline 岁 & 12 & & & & & & & & & & & & & & & & 1 & 1 & 3 & & & & & & & & & & & & & & 5 \\
\hline 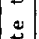 & 13 & & & & & & & & & & & & & & & 2 & 2 & - & 3 & 5 & & & & & & & & & & & & & 12 \\
\hline 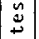 & 14 & & & & & & & & & & & 1 & 1 & 2 & - & - & - & 2 & - & - & 6 & 4 & 2 & & 3 & & & & & & & & 21 \\
\hline$\therefore$ & 15 & & & & & & & 1 & - & - & & - & - & 1 & 1 & - & 1 & - & 2 & 4 & 4 & 1 & - & & -2 & 2 & 1 & & & & & & 18 \\
\hline$\therefore$ & 16 & & & & & & & & 1 & - & & - & - & - & 2 & 1 & 3 & 1 & 2 & 1 & 1 & 3 & - & & 11 & 1 & & & & & & & 17 \\
\hline$\stackrel{\mathscr{a}}{10}$ & 17 & & & & & & & & & & & & & & & 4 & - & 1 & 2 & 4 & & & & & & & & & & & & & 11 \\
\hline 西 & 18 & & & 1 & - & & - & - & - & - & & - & - & 1 & 2 & 1 & - & 1 & 1 & 5 & 1 & 1 & 1 & & - - & - & - & 1 & - & - & 2 & & 18 \\
\hline 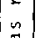 & 19 & & & & & & & & & & & 1 & 1 & - & 1 & - & - & - & 2 & 2 & - & - & 1 & & & . & & & & & & & 8 \\
\hline च & 20 & & & & & & & & & & & & & & & 2 & 2 & 3 & 2 & 1 & & & & & & & & & & & & & 10 \\
\hline 髡 & 21 & & & & & & & 1 & - & - & & - & - & 3 & - & - & 1 & 1 & 1 & 2 & & & & & & & & & & & & & 9 \\
\hline$\stackrel{8}{2}$ & 22 & & & & & & & & & & & 2 & - & 1 & - & 1 & - & - & - & - & 1 & & & & & & & & & & & & 5 \\
\hline 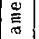 & 23 & & & & & & & & & 1 & & - & - & - & - & - & - & 1 & - & & & & & & & & & & & & & & 2 \\
\hline 5 & 24 & & & & & & & & & & & & 2 & - & - & - & - & - & - & & & & & & & & & & & & & & 2 \\
\hline & 25 & & & & & & & & & & & 1 & - & - & - & - & - & - & - & & & & & & & & & & & & & & 1 \\
\hline & 26 & & & & & & & & & & & & & & & & - & 1 & - & & & & & & & & & & & & & & 1 \\
\hline & 27 & & & & & & & & & & & & & & & & -1 & - & - & & & & & & & & & & & & & & - \\
\hline & 28 & & & & & & 1 & - & - & - & & - & - & - & - & - & - & - & - & & & & & & & & & & & & & & 1 \\
\hline & TOTAL & - & - & $\overline{1}$ & - & & 1 & 2 & 1 & 1 & & 5 & 4 & 8 & 7 & 713 & 15 & 18 & 24 & 29 & 13 & 9 & 4 & & $\overline{43}$ & 3 & 1 & $T$ & - & - & 2 & & 166 \\
\hline & & $N_{-}=$ & $58 ; 8$ & $=3$, & $43 \mathrm{~m}$ & nm ; & $s=$ & 2, & 56 & $\mathrm{~mm}$ & ; $\Sigma$ & $x=$ & $t=-$ & 199 & & & & & & $1+=$ & $=90 ;$ & $; 8$ & $=$ & 2,92 & $2 \mathrm{mr}$ & $\mathrm{nm}$; & $S=$ & 2,2 & $26 \mathrm{~m}$ & n: $\boldsymbol{\Sigma}$ & $x=$ & 263 & \\
\hline
\end{tabular}


Tabela 7. Risco anual de infecção tuberculosa (Ri) em escolares com 6 - 9 anos de idade, segundo antecedente vacinal e segundo o método utilizado para o cálculo da prevalência de infectados. Alunos das Escolas Municipais da zona leste da Cidade de São Paulo - 1988.

\begin{tabular}{|c|c|c|c|c|c|c|}
\hline $\begin{array}{l}\text { Antecedente } \\
\text { vacinal }\end{array}$ & $\begin{array}{l}\text { Métod } \\
\text { de cálcL }\end{array}$ & & $\begin{array}{l}\text { Ne de escolares } \\
\text { testados }\end{array}$ & $\begin{array}{l}\text { Média } \\
\text { de Idade }\end{array}$ & $\begin{array}{l}\text { Prevalência de } \\
\text { infeç̧ăo }(\%)\end{array}$ & $\begin{array}{l}\mathrm{Ri} \\
(\%)\end{array}$ \\
\hline $\begin{array}{l}\text { Vacinados no primeiro } \\
\text { trimestre" }\end{array}$ & Dam e Hitze & original & 3.270 & 6 anos e 8 meses & 2,32 & 0,35 \\
\hline $\begin{array}{l}\text { Vacinados no primeiro } \\
\text { trimestre" }\end{array}$ & Dam e Hitze & modificado & 3.270 & & 2,97 & 0,45 \\
\hline $\begin{array}{l}\text { Vacinados após o primeiro } \\
\text { trimestre*" }\end{array}$ & Dam e Hitze & original & 4.110 & 8 anos e 5 meses & 3,04 & 0,37 \\
\hline $\begin{array}{l}\text { Vacinados após o primeiro } \\
\text { trimestre*" }\end{array}$ & Dam e Hitze & modificado & 4.110 & & 4,04 & 0,49 \\
\hline $\begin{array}{l}\text { Vacinados nos primeiros } \\
\text { dois anos }\end{array}$ & Bhattacharya & & 10.855 & 7 anos e 8 meses & 4,15 & 0,55 \\
\hline Năo vacinados & Reatores fortes & $(\geq 10 \mathrm{~mm})$ & 601 & 7 anos e 11 meses & 4,48 & 0,58 \\
\hline
\end{tabular}

- Com $1 / 2$ dose de vacina

* Com dose plena de vacina
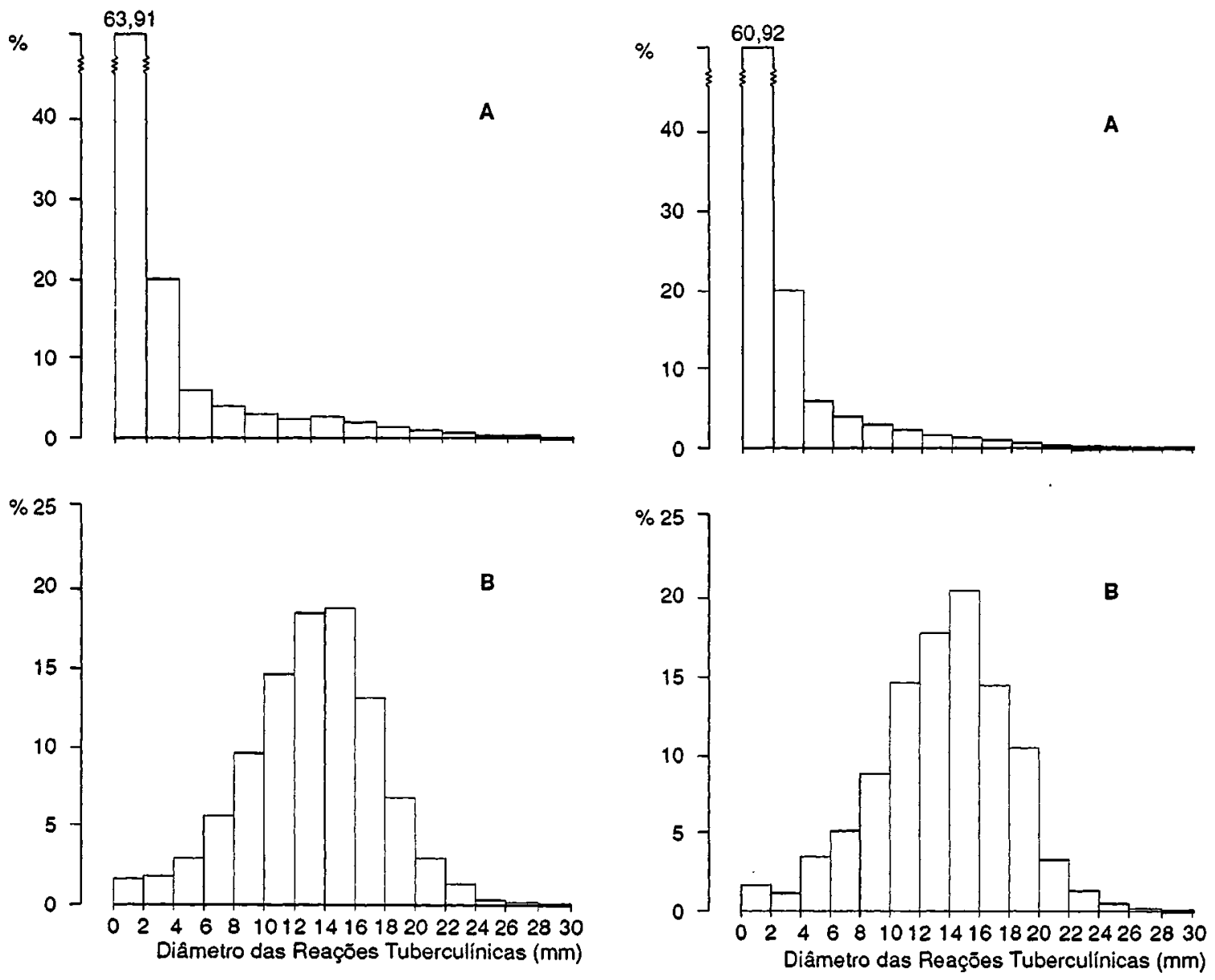

Figura 1. Distribuição das reaçōes ao primeiro teste de Mantoux (A) e ao segundo Mantoux (B) nos escolares primovacinados com BCG no primeiro trimestre de vida. Zona leste de S. Paulo, 1988.

Figura 2. Distribuição das reaçōes ao primeiro teste de Mantoux (A) e ao segundo Mantoux (B) nos escolares primovacinados com BCG depois do primeiro trimestre de vida. Zona leste de S. Paulo, 1988. 

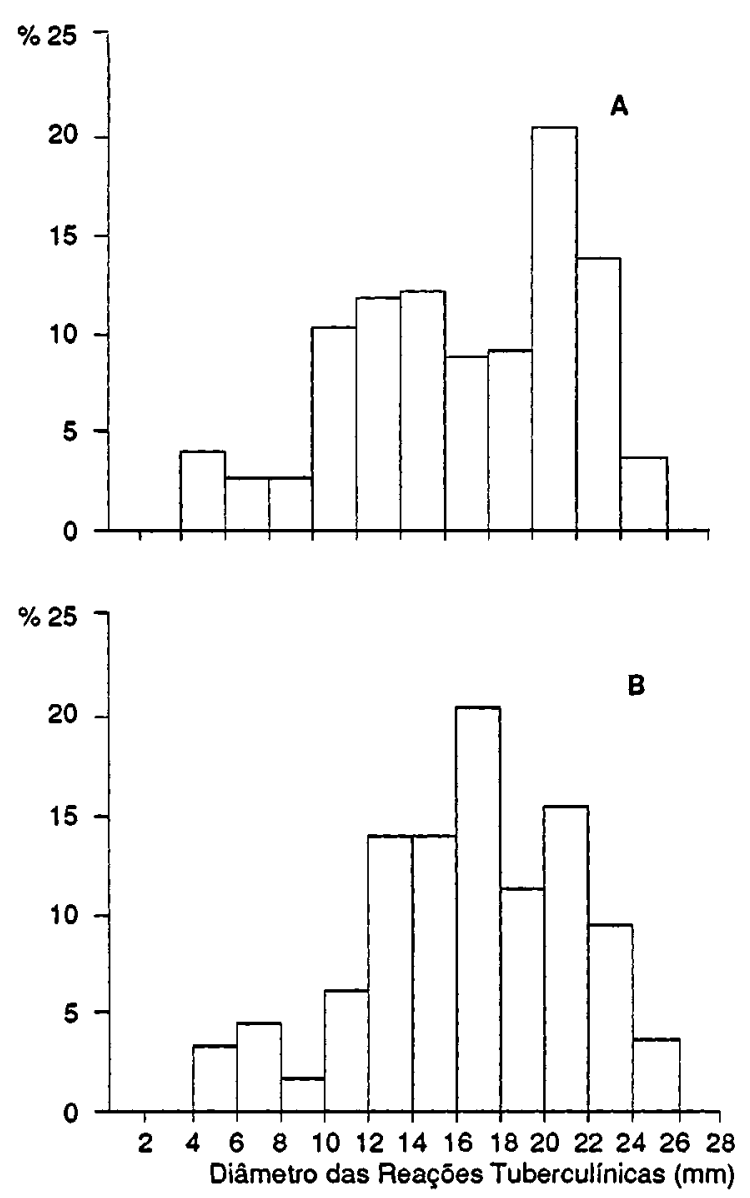

Figura 3. Perfil tuberculínico dos infectados identificados pelo método original de Dam e Hitze $(A)$ e pelo método modificado $(B)$ em escolares vacinados com BCG no primeiro trimestre de vida. Zona leste de S. Paulo, 1988.

populações, segundo os critérios utilizados para a identificação e contagem dos infectados, em confronto com dois referenciais disponiveis para a área em questão.

\section{Discussão}

Inquéritos tubcrculínicos são inviávcis $\mathrm{cm}$ amostras domiciliares probabilísticas devido aos elevados custos, razão pela qual se tem dado preferência, no Brasil, a populaçõcs escolares.

As perdas observadas entre a aplicação e a leitura do Mantoux (8 a 10\%), provavelmente devidas ao absenteísmo, foram semelhantes às observadas em inquéritos realizados anteriormente na área. A perda da primeira para a segunda etapa (17\%), mais acentuada, parece representar o acrés- cimo de outro fator - evasão ou transferência - o que poderia significar algum tipo de seleção a favor dos que permaneceram nas escolas ${ }^{2}$. A comparação entre os dois subgrupos (8.329 submetidos aos dois testes e os 3.126 testados apenas uma vez) não revelou diferença significante quanto à composição por sexo e idade; e a proporção de portadores de cicatriz vacinal foi idêntica em ambos, bem como a prevalência de reatores fortes*. Quanto à comparação entre vacinados e não vacinados, em termos de prevalência de infecção, ela é questionável se a cobertura vacinal for muito elevada, admitindo-se que os não vacinados possam representar um grupo populacional sob maior risco de infecção tuberculosa ${ }^{21}$.

Na população estudada, a composição por sexo e idade foi semelhante nos dois subgrupos, o mesmo ocorrendo com a distribuição segundo subregiơes de residência ${ }^{3}$. Além do mais, uns e outros estavam inseridos no contexto escolar, freqüentando as mesmas escolas e o mesmo periodo do ano letivo.

Contudo, não se pode afastar a possibilidade de riscos de infecção difcrentes entre os dois subgrupos, nos anos que antecederam a entrada na escola, uma vez que não se pesquisou a procedência e o tempo de residência na área.

A análise dos dados das Tabelas 1 e 2 mostra que o incremento causado pcla revacinação foi muito expressivo nas reações nulas ou fracas ao primeiro tcste, em comparação com o incremento observado nas reações mais fortes.

O perfil tuberculinico pré vacinal (Figuras $1 \mathrm{~A}$ e $2 A)$ sugere uma distribuição assimćtrica à direita que na verdade esconde no seu bojo três distribuições normais, como já foi demonstrado ${ }^{4,26}$. Evidentemente a prevalência de infecção tuberculosa, calculada pelo critério das normas técnicas, seria superestimada ${ }^{3}$, principalmente na subpopulação vacinada na segunda idade, inoculada com o dobro da quantidade injetada na primeira idade.

Por sua vez o perfil pós vacinal delincia uma distribuição normal (Figuras $1 \mathrm{~B}$ e $2 \mathrm{~B}$ ), como seria de esperar ${ }^{22}$, para qual os reatores nulos ou fracos ao primeiro teste contribuiram com maior ênfase.

A distribuição das diferenças entre as duas leituras nos infectados, assim identificados, (Tabela 3) revela que apesar de o número de observações abaixo da diagonal ter sido apenas $27 \%$ maior do que o número acima dela, a magnitude $(\Sigma \mathrm{x})$ dos desvios negativos foi quase o dobro da magnitude dos desvios posilivos, c a média das diferenças foi menor do que zero $(-0,64 \mathrm{~mm})$, contrariando a expectativa de uma ligeira predominância positiva

* Dados inćditos à disposição dos interessados. 
devida ao efeito "boostcr" do teste tuberculínico $^{14,15}$. Todavia, com o balizamento dos infectados localizados acima da diagonal levando em conta, em cada linha, o produto do númcro pela amplitude dos desvios negativos, os resultados foram mais coerentes com o conhecimento disponivel a respeito do erro experimental do teste padronizado ${ }^{7,17}$.

Assim realizada, a contagem dos indivíduos acima da diagonal aumentou de 26 para 47 (+ $80 \%$ ), porém a magnitude dos desvios positivos superou os negativos em apenas 14\% (Tabcla 4), e a média da distribuição das diferenças se tomou discretamente positiva $(0,16 \mathrm{~mm})$ com desviopadrão $3,16 \mathrm{~mm}$. Vale ressaltar que a margem de erro experimental implícita nesse procedimento se manteve próxima dos limites de $\pm 6 \mathrm{~mm}$, cstabclecidos em dois inquéritos consecutivos, sem vacinação intermediária ${ }^{13}$.

O perfil tuberculínico dos infectados, identificados pelo procedimento alternativo, mostrou-se mais aproximado à distribuição normal do que o perfil obtido com a proposta original (Figs. $3 \mathrm{~A} \mathrm{c}$ B). O mesmo deu-se com os infectados do grupo vacinado na segunda idadc: a distribuição das diferenças entre as leituras apresentou média negativa, superioridade de $18 \%$ no númcro de negativos, a qual alcançou $100 \%$ em termos de magnitude dos desvios.

Tal como no grupo anterior, a contagem dos infectados, tomando por base a amplitude dos desvios (Tabela 6), além de amenizar a diferença entre as somatórias dos desvios positivos e negativos, revelou uma média positiva próxima de zero.

Os resultados obtidos segundo o momento da primovacinação e conforme diferentes critérios de contagem dos infectados, comparados com duas estimativas disponiveis, são apresentados na Tabela 7. Um dos referenciais $-4,15 \%$ - foi estimado em 10.855 escolares dessa população (vacinados no primciro ano de vida) a partir da decomposição gráfico-matemática do respectivo perfil tuberculínico ${ }^{4}$. Esse método, aplicado pela primeira vez em vacinados, Lora utilizado com sucesso $\mathrm{cm}$ população contaminada por micobactérias atípicas 26, 27, 28.

O outro refcrencial $-4,48 \%$ - foi estimado $\mathrm{cm}$ apenas 602 escolares não vacinados, com as ressalvas apontadas, interpretando-se como infectados os reatores com 10 ou mais milímetros de diâmetro ${ }^{12}$.

A transformação das prevalências $\mathrm{cm}$ riscos de infecção, por corrigir as difcrenças de idade, é necessária para cfetuar o confronto entre os critćrios (original e alternativo) e cntre os momentos da primovacinação (primeira e segunda idades). Isto posto, os riscos de infecção estimados pelos dois critérios e segundo os momentos $(0,35 \%, 0,37 \%$, $0,45 \%$ e $0,49 \%$ ) foram comparados entre si e com a média dos referenciais disponiveis $(0,55 \%)$. As difcrenças entre critérios $e$ idades e destes com o refcrencial não foram significantes ( $P>0,05$ ).

Em suma, o momento da vacinação no primeiro ano de vida parece não ter influído na estimativa do risco de infeç̧ão, ao passo que a modificação introduzida no critério de contagem dos infectados, além de melhorar o scu perfil tuberculinico, forneceu resultados mais próximos dos referenciais.

Pelo visto, ao contrário da expectativa de Dam e Hilzc ${ }^{5}$, o método é aplicável em vacinados com dose plena e por isso recomendável para o acompanhamento da cvolução do risco de infecção tuberculosa $\mathrm{cm}$ circunstâncias semelhantes às da árca estudada.

ARANTES, G. R. et al. [Determining the risk of tuberculosis infection in BCG-vaccinated populations]. Rev. Saúde públ., S.Paulo, 26: 96-107, 1992. The revaccination of schoolchildren can restore the residual allergy induced by vaccination in the first years of life but can not modify the allergy resulting from a natural infection. So revaccination in this population should indicate the group infected by the Koch bacilli. To assess the applicability of these assumptions in estimating the risk of tuberculosis infection in regions with high BCG coverage a study was undertaken on schoolchildren between 6 and 9 years of age who were attending the municipal schools in the cast zone of S. Paulo City, in the course of the first semester of 1988. Of 11,455 who were vaccinated only 7,470 were tested with PPD, revaccinated and retested ten weeks later; 3,314 of these were vaccinated in the first trimester of life with a half dose and 4,156 received a full dose at later ages $(75 \%$ during the first year, $20 \%$ during the second and 5\% during the third). In comparing the results pre and post vaccination by correlation table, the calculation of infection was made according to the criteria of the original method and to the modifications made by the authors under separate cover for those vaccinated in the first trimester and those vaceinated later. The risk of infection was $0.35 \%$ and $0.37 \%$, respectively, for the original model and $0.45 \%$ and $0.49 \%$ for the modified model. The referencial was $0.55 \%$. The difference between model and age or with the referencial was not significant $(p>0.005)$. Data suggest that the method is applicable to estimate the risk of tuberculosis infection in schoolchildren vaccinated with a full dose of BCG during the first year of life.

Keywords: Tuberculosis, prevention and control. BCG vaccine. Population surveillance.

\section{Referências Bibliográficas}

1. AMERICAN THIORACIC SOCIETY. The tuberculin skin test. Amer. Rev, resp. Dis., 124: 356-63, 1981. 
2. ARANTES, G. R.; NARDY, S. M. C.; NASSAR, J. Estudo sobre a evolução do risco de infecção tuberculosa em area com elevada cobertura por BCG. Rev. Saúde públ., S. Paulo, 19: 95-107, 1985.

3. ARANTES, G. R.; NARDY, S. M. C.; NOGUEIRA, P. A.; BELLUOMLNI, M.; WLILER, R. M. G. Influência do BCG aplicado no primeiro ano de vida no perfil tuberculínico à idade escolar. Medicina, Ribeirão I'reto, 23: 232-41, 1990

4. ARANTES, G. R.; NARDY, S. M. C.; NOGUEIRA, P. A.; BELLUOMINI, M.; WEILER, R. M. G. Estimativa da prevalência de infecção tuberculosa em escolares vacinados com BCG, por meio do método de Bhattacharya. Rev. Saúde públ., S. Paulo, 25: 112-20, 1991.

5. DAM, H. G. ten \& HITZE, K. L. Determining the prevalence of tuberculosis infection in population with non specific sensibility. Bull. Wld Hlth Org., 58: 475-83, 1980.

6. GESER, A.; ROY, L. A.; BLOCHER, C. Direct BCG vaccination in Burandi, Dahomey and Mauritânia: a study of local complication. Bull. Wld Hllh Org., 35: 609-32, 1966.

7. GULD, J. Quantitative aspects of the intradermal tuberculosis tests in humans. Acta tuberc. scand., 28: 223-34, 1953.

8. GULD, J.; MAGNUS, K.; TOLDERLUND, K.; BIERNGSORENSEN, K.; EDWARDS, P. Q. Suppurative lymphadenitis following intradermal BCG vaccination of the newbom. Brit. med. J., 2: 1048-54, 1955.

9. GULD, J.; WAALER, H.; SUNDARESAN, T. K.; KAUF MANN, P. C.; DAM, H. G. ten. The duration of BCG induced tuberculin sensitivity in children, and its irrelevance for revaccination. Bull. Wld llllh Org., 39: 82936, 1968 .

10. MAGNUS, K. Effect of intrademal tuberculin tests on BCG - induced allergy. Bull. Wld Hllh Org., 17: 249 54, 1957.

11. MNTSTÉRIO DA SAÚDE. Divisão Nacional de Pncumologia Sanitária. Manual de Normas Técnicas para o Controle da Tuberculose. Brasília, 1979.

12. MINISTÉRIO DA SACDE. Divisão Nacional de Pncumologia Sanitária. Manual de Normas Técnicas para o Con. trole da Tuberculose. $3^{2}$ ed. rev. Brasilia, 1988.

13. NARAIN, R.; NAIR, S.S.; CHANDRASE KHAR, P.; RAO, G. R. Problems connected with estimating the incidence of tuberculosis infection. Bull. Wld Hlih Org., 34: 605-22, 1966.

14. NARAIN, R.; NAIR, S.S.; RAO, G. R.; CHANDRASE KHAR, P.; LAL, P. Enhancing of tuberculin allergy by previous tuberculin testing. Bull. Wld Illth Org., 34: 623-38, 1966.
15. NARAIN, R. Interpretation of the repeat tuberculin testing. Tubercle, London, 49(1): 92-103, 1968.

16. NARAIN, R. \& VALLISHAYEE, R. S. Post vaccination allergy after three intervals of time: preliminary report. Bull. int. Un. against Tuberc., 51(1): 231-7, 1976.

17. NISSEN-MEYER, S.; HOUGEN, A.; EDWARDS, P. Experimental error in the determination of tuberculin sensitivity. Publ. Hlih Rep., 66: 561-9, 1951.

18. NYBOE, J. The efficacy of the tuberculin test. Bull. Wld Hlih Org., 221: 5-37, 1960.

19. NYBOE, J. The immediate effects of BCG revaccination. Bull. Wlalllih Org., 41: 63-73, 1969.

20. OLAKOWSKI, T. \& MARDON, K. The restorative influence of repeated uberculin testing on tuberculosis sensitivity in BCG vaccinated schoolchildren. Bull. Wld Hlth Org., 45: 649-55, 1971.

21. ORGANIZACION PANAMERICANA DE LA SALUD. Control de tuberculosis en America Latina: manual de normas y procedimientos para programas integrados. Washington, D. C., 1979. (Publicacion Científica, 376).

22. PALMER, C. E. BCG vaccination and tuberculin allergy. Lancet, 262: 935-40, 1952.

23. PALMER, C. E. \& LONG, M. W. Effects of infection with atypical mycobacteria on BCG vaccination and uberculosis. Amer. Rev. resp. Dis., 94: 553-68, 1966.

24. PRANDI, R.; PIERUCCI, A. F.; MENDES, A. M. T. A definição do voto. São Paulo, 1985. [Apresentado no $9^{\circ}$ Encontro Anual da Associação Nacional de PósGraduação e Pesquisas em Ciências Sociais, Águas de S. Pedro, S. P., 1985].

25. REIS, F. J. C. Alergia tuberculínica e cicatriz vacinal em lactentes que tomaram a vacina BCG injetável quando recém-nascidos. J. Pediat., 52(1/2): 23-8, 1982.

26. RUFFLNO-NETTO, A.; SANCHES, O.; ARANTES, G. R. Aplicação do método de Bhattacharya na análise de resultados do teste tuberculínico. Rev. Saúde públ., S. Paulo, 11: 322-9, 1977.

27. SANCIIES, O. Classificação das pessoas na prova tuberculínica: aplicação de um modelo estaústico quando a distribuição de freqüĉncias é uma mistura de componentes nomais. Rev. Saúde públ., S. Paulo, 10: 285-9, 1976.

28. SANCHES, O.; ARANTES, G. R.; RUFFINO-NETTO, A: Probabilidade de classificação errada ao teste tuberculínico segundo critério em vigor. Rev. Div. Nac. Tuberc., 21(83): 288-95, 1977.

Recebido para publicação em 1/8/1991 Aprovado para publicaçāo em 19/11/1991 